\title{
0 Efeito do Mercosul Sobre a Comercialização de Novos Produtos ${ }^{*}$
}

\author{
Erik Figueiredo ${ }^{\dagger}$ \\ Alexandre Loures ${ }^{\ddagger}$
}

Sumário: 1. Introdução; 2.0 que os dados têm a dizer?; 3. Especificação econométrica; 4. Resultados; 5. Conclusão.

Palavras-chave: Equação Gravitacional, Margem Extensiva de Comércio, Mercosul.

Códigos JEL: $\quad$ C33, C55, F14, F15.

Este artigo utiliza um banco de dados com elevado nível de desagregação com intuito de mensurar o impacto do Mercosul sobre a criação de novas relações comerciais. Para tanto, será utilizada a definição de margem extensiva de comércio contida em Baldwin \& Di Nino (2008), qual seja: o número de produtos que não pertenciam a pauta de exportação antes da assinatura do acordo bilateral. Os resultados indicam que o Mercosul possui um impacto positivo sobre a probabilidade de uma nova categoria de produtos ser comercializada (em torno de $1,87 \%)$. Observou-se também que Paraguai e Uruguai foram os países mais beneficidados com o acordo.

This article uses a database with a high level of disaggregation in order to measure the impact of Mercosur on the improvement of the new business relationships. So that, it will consider the extensive margin definition of trade developed by Baldwin $\varepsilon$ Di Nino (2008), namely: the number of products that did not belong to export products before signing the bilateral agreement. The results indicate that Mercosur has a positive impact on the probability of a new class of product to be traded (around $1.87 \%)$. It was also noted that Paraguay and Uruguay were the most benefit countries with the agreement.

\footnotetext{
*Agradecemos a um parecerista anônimo isentando-o de eventuais erros e/ou omissões cometidos ao longo do texto. Também somos gratos ao suporte financeiro do CNPq por meio do projeto 441473/2014-8.

${ }^{\dagger}$ Universidade Federal da Paraíba (UFPB), Centro de Ciências Sociais Aplicadas (CCSA), Programa de Pós-Graduação em Economia (PPGE). Cidade Universitária, Campus I, João Pessoa, PB, Brasil. CEP 58051-900. Email: eaf igueiredo@gmail .com

†Universidade Federal da Paraíba, Centro de Ciências Sociais Aplicadas (UFPB/CCSA), Departamento de Economia. Cidade Universitária, Campus I, João Pessoa, PB, Brasil. CEP 58051-900. Email: alexandre.loures@ymail .com
} 


\section{INTRODUÇÃO}

Os Regional Trade Agreements (RTAs) têm se constituído como uma das principais ferramentas de facilitação do comércio bilateral. Para se ter uma ideia, em 1992 haviam apenas 28 RTAs em vigência. Esse número passou para 273 em 2015, um crescimento de 875\% (ver Figura 1). Uma das razões para esse comportamento reside na constatação de que RTAs impulsionam em quase cinco vezes o comércio entre os seus membros (Baier \& Bergstrand, 2007). Ademais, seus efeitos positivos também são registrados sobre outros fluxos econômicos, tais como, FDI e migração (Baltagi, Egger \& Pfaffermayr, 2008; Orefice, 2015).

Recentemente, Baier, Bergstrand \& Feng (2013) chamaram a atenção para duas importantes fontes de heterogeneidade dos efeitos dos RTAs. Primeiro, registram-se diferenças de acordo com o nível de profundidade. Regra geral, acordos mais profundos, como a união aduaneira, possuem um efeito mais expressivo sobre o fluxo comercial quando comparadas, por exemplo, aos acordos de comércio preferencial uni- e multi-laterais (PTAs). Segundo, os RTAs tendem a apresentar um efeito contemporâneo mais forte sobre a margem intensiva de comércio - média do valor dos produtos já comercializados no mercado internacional - do que sobre a margem extensiva - número de produtos comercializados.

A constatação empírica dos efeitos heterogêneos dos RTAs nas margens comercializáveis estão em consonância com as predições das teorias modernas de comércio. Os modelos de Melitz (2003) e Chaney (2008), por exemplo, consideram que apenas as firmas mais produtivas são capazes de suplantar um determinado nível de custo fixo de entrada no mercado internacional. Nesse sentido, caso o RTA reduza esse custo, haverá um maior número de empresas aptas a direcionar seus produtos para o mercado externo, aumentando a margem extensiva de comércio. De outro lado, uma queda no custo variável estimularia o comércio de bens já comercializados, ou seja, impactaria na margem intensiva de comércio. ${ }^{1}$

Figura 1. Acordos em vigor por ano (acumulado).

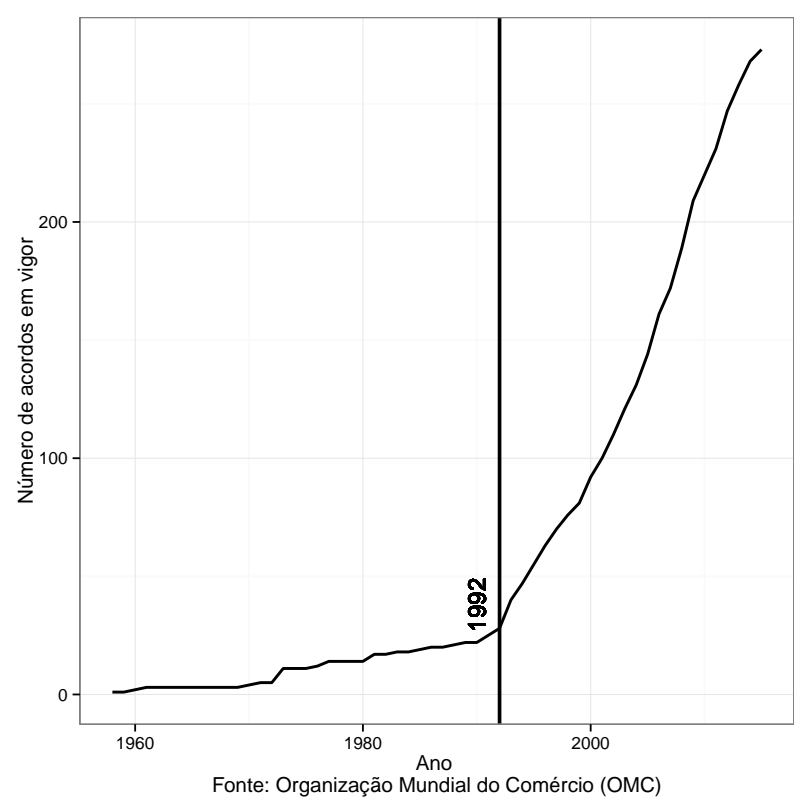

\footnotetext{
${ }^{1}$ A discussão sobre os efeitos dos custos sobre a margem intensiva de comércio é controversa. Para maiores detalhes, ver Dutt, Mihov \& Van Zandt (2013).
} 
Recentemente, Baldwin \& Di Nino (2008) contestaram a definição tradicional de margem extensiva de comércio. Para esses autores, a margem extensiva deve ser entendida como o número produtos que não faziam parte da pauta de exportação antes da assinatura do RTA. Esse resultado ficou conhecido como a "hipótese dos novos produtos". Sob ela, a adoção de uma moeda comum operaria da mesma forma que uma união aduaneira, reduzindo os custos comerciais e estimulando o comércio de novos produtos entre seus membros. De fato, Baldwin \& Di Nino (2008) demonstram que o uso comum do Euro estimula a exportação de novos produtos ao invés de simplesmente aumentar o volume de comercialização dos bens que já eram comercializados antes da integração econômica, ou seja, os acordos bilaterais de comércio possuem um efeito mais expressivo sobre o novo conceito de margem extensiva.

Diante do exposto, este estudo pretende investigar qual o impacto do Mercosul sobre a entrada de novos produtos na pauta de exportação dos países membros. Tal análise torna-se possível uma vez que o Mercosul encontra-se na segunda fase de uma integração econômica, união aduaneira, em que se eliminam ou se reduzem substancialmente todos os direitos aduaneiros e restrições comerciais, no intercâmbio de produtos originários da região, bem como estabelece uma Tarifa Externa Comum (TEC) ${ }^{2}$ e uma mesma política comercial, em relação a produtos originários de terceiros países.

Para alcançar esse objetivo, o paper adotará os seguintes procedimentos metodológicos:

(i) Utilização de uma base de dados com um alto nível de desagregação permitindo captar a comercialização de novos produtos;

(ii) Assume-se como regressando uma variável binária em que um representa fluxo de comércio positivo enquanto zero caracteriza a ausência de comércio ou censura aos dados;

(iii) Um grupo de tratamento composto pelos membros plenos do Mercosul (Brasil, Argentina, Paraguai, Uruguai e Venezuela) e dois grupos de controle: um formado por Bolívia, Chile e Peru e o outro por Alemanha, Estados Unidos e Holanda; e

(iv) o uso do estimador Logit com erros robustos à heterocedasticidade.

Além desta Introdução este artigo está organizado da seguinte forma: Na seção 2 objetiva-se identificar, isso é, ratificar a hipótese dos bens recém comercializados através de uma análise dos dados. Detalhes sobre a especificação econométrica e a estimação são mostrados na seção 3. Na seção 4 apresentam-se e se discutem os resultados. Na seção 5 são feitas as considerações finais.

\section{O QUE OS DADOS TÊM A DIZER?}

Os dados utilizados neste artigo foram extraídos da Base pour l'Analyse du Commerce International $(\mathrm{BACI})^{3}$, do Centre D'Estudes Prospectives Et D'Informations Internationales (CEPII). Esse conjunto de dados é a base sobre o comércio internacional que abrange o maior número possível de países e cuja classificação de agregação é denominada de Sistema Harmonizado de quatro dígitos (HS4), ${ }^{4}$ em substituição à Classificação Padrão do Comércio Internacional (SITC) $)^{5}$ de quatro dígitos. O Sistema Harmonizado, criado em 1988, caracteriza-se como uma classificação internacional de mercadorias baseada em códigos e suas respectivas descrições cujo objetivo é promover o aprimoramento do comércio internacional permitindo

\footnotetext{
${ }^{2}$ Caracteriza-se como uma tarifa aduaneira comum a todos os membros da Área Livre de Comércio (ALC) e válida para importações provenientes de fora do bloco e cujo objetivo é aumentar a eficiência intrabloco.

${ }^{3}$ Anualmente os países reportam à Divisão de Estatística das Nações Unidas os respectivos fluxos comericiais bilaterais e, assim, essa divisão monta a base de dados denominada de Commodities Trade Statistic database (COMTRADE), representando mais de 95\% do comércio mundial e sendo a única fonte de informação para a elaboração da BACI. Para mais detalhes veja Gaulier \& Zignago (2010).

${ }^{4}$ Harmonized System 4.

${ }^{5}$ Abreviação em inglês para Standard International Trade Classification (SITC).
} 
comparações e análises estatísticas entre os países bem como facilitar as negociações internacionais (através da padronização das informações).

Contudo, essa base possui uma desvantagem em relação ao nível de agregação causando assim dois importantes problemas. Primeiramente, uma vez que cada uma dessas categorias abrange uma ampla gama de produtos ocorrerá que uma mesma categoria abrigará tanto bens que já eram comercializados antes da implementação de um RTA bem como bens que não eram comercializados, inviabilizando mensurar o impacto total de um acordo comercial regional sobre a margem extensiva. Segundo, como as grandes economias, na maioria das vezes, comercializa uma ampla gama de produtos, então, após a assinatura de um RTA o impacto sobre a margem extensiva será menor comparativamente a uma pequena economia. Por exemplo, ao analisarem o efeito do uso do Euro sobre a margem extensiva Baldwin \& Di Nino (2008) verificaram que o impacto foi maior para as menores economias, denominando esse problema de viés de agregação. Diante do exposto, a base ideal seria em nível de firma, o que permitiria capturar o impacto total sobre a margem extensiva, porém essa não está disponível para o Mercosul.

Dessa forma, considerando os dados do Mercosul em que, em 1991, o número de categorias comercializadas por cada um dos membros plenos foi de: i) Brasil, 1.043; ii) Argentina, 934; iii) Paraguai, 249; iv) Uruguai, 505; e v) Venezuela, 584. Com isso, é de se esperar que após a implementação do Mercosul a margem extensiva brasileira tenha sofrido o menor impacto, comparativamente aos demais países. Assim, objetivando verificar essa hipótese analisaram-se os resultados para 2010 e, conforme a Figura 2, nota-se que os valores para Brasil e Paraguai foram respectivamente, 1.150 (10,3\%) e 535 $(114,9 \%)$, corroborando com a percepção inicial. Contudo, a maior variação foi para a Bolívia (Estado associado) com 348,7\% de aumento, pois antes do Mercosul haviam 119 categorias comercializadas e depois essas totalizaram 534. Esses resultados demonstram que para o comércio internacional em 2010 a margem extensiva do Mercosul variou entre 10,3\% à 348,7\%, considerando 1991 como o ano base, isso é, evidenciaram o tamanho significativo da margem extensiva (Figura 2).

Todavia, como a informação central ${ }^{6}$ para a hipótese dos bens recém comercializados é a queda no número de "zeros" na matriz de comércio dos membros do Mercosul verificar-se-á através de uma abor-

Figura 2. Evolução no número de bens comercializados.

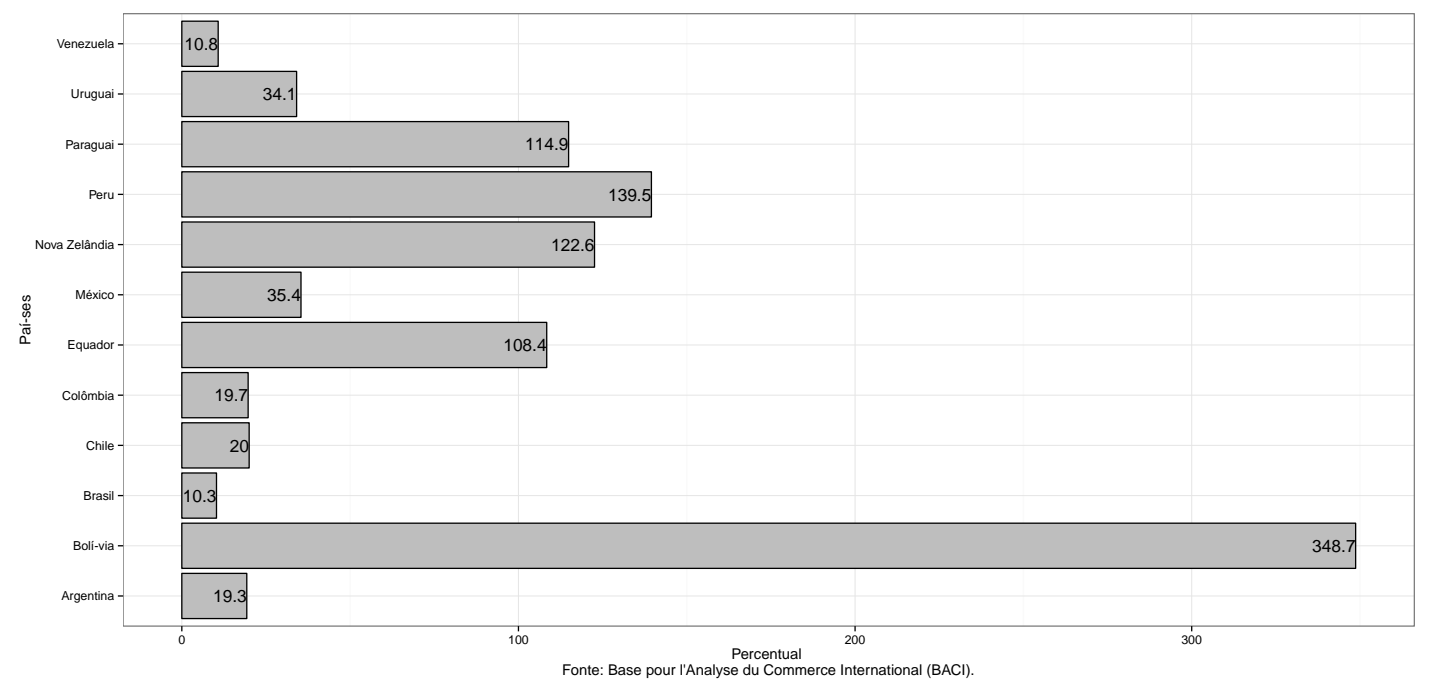

\footnotetext{
${ }^{6}$ Segundo Baldwin, Di Nino, Fontagné, De Santis \& Taglioni (2008, p.86) “a hipótese dos bens recém comercializados está totalmente alicerçada sobre a redução no número de 'zeros' no fluxo de comércio."
} 
dagem de diferenças-em-diferenças ${ }^{7}$ a queda do fluxo de comércio contabilizado como "zero" entre os membros plenos do Mercosul em relação ao comércio entre membros e não-membros. Segundo Baldwin \& Di Nino (2006) a primeira diferença caracteriza-se como sendo uma análise de estática comparativa na mudança dos "zeros" entre os períodos anteriores e posteriores a implementação do RTA enquanto a segunda diferença representa uma comparação entre o fluxo de comércio bilateral dos membros do Mercosul e dos não-membros. Se a hipótese dos bens recém-comercializados estiver certa o que se verificará é uma queda maior nos "zeros" entre os membros do Mercosul do que entre esses e os não-membros.

Contudo, como destacado por Baldwin \& Di Nino (2008) embora essa informação seja um bom indicativo para a evolução dos "zeros" na matriz de comércio ela pode ser espúria, uma vez que a queda no número de "zeros" pode ter como consequência outros motivos do que única e exclusivamente a implementação de um RTA. Por exemplo, mudanças nos hábitos de consumo dos agentes econômicos nos países membros de um RTA podem induzir essas nações a importarem mais de todos os demais países. Então, é de suma importância analisar também a evolução dos "zeros" no fluxo comercial entre os não-membros e os membros bem como o comércio exclusivamente entre os não-membros.

Por simplicidade e padronização de agora em diante os fluxos de comércio entre membros e nãomembros $^{8}$ serão representados da seguinte forma: SUR11, SUR10, SUR00 e SUR01 em que o primeiro numeral representa o país de origem enquanto o segundo o de destino. Assim, SUR11 quer dizer que o exportador é um membro do Mercosul assim como o importador, SUR10 o exportador é um membro do Mercosul e por sua vez o importador é um não-membro, SUR00 tanto o exportador quanto o importador são não-membros do Mercosul e SUR01 o exportador é um não-membro e o importador é membro do Mercosul.

Percebe-se pela Figura 3 que os "zeros" das matrizes comerciais de todos os fluxos comerciais (SUR11, SUR10, SUR01 e SUR00) apresentaram uma tendência de queda desde o fim da década de 80, o que pode estar relacionado ao processo de globalização, porém o comportamento da variável SUR11 destoa das demais. Após a constituição do Mercosul o que se nota é uma forte redução no número de "zeros", principalmente a partir de 1992, resultando numa queda de $30 \%$ em 1995 . O comportamento dos períodos seguintes é marcado por alguns "picos" e "vales" atingindo em 2005 o maior nível de redução (33\%). O grupo de controle para essa variável, SUR00, também apresentou um comportamento de queda no período, entretanto, mais suavizado (embora em anos recentes tem sido maior do que o do Mercosul).

Como um teste de robustez analisou-se o comportamento da variável SUR01 verificando, Figura 3, que assim como as demais, essa curva apresentou uma tendência de queda, porém foi o movimento mais suavizado de todas as curvas, demonstrando que foi o Mercosul e não motivos outros que provocaram a redução no número de "zeros". Já com relação a variável SUR10 nota-se que o Mercosul impulsionou também as exportações dos membros para os não-membros.

Importante destacar que uma parcela dos "zeros" na BACI são dados censurados, pois as transações inferiores a US\$1.000 não são contabilizadas no comércio internacional e, dessa forma, os "zeros" podem representar tanto a ausência de comércio bem como censura aos dados.

\section{ESPECIFICAÇÃO ECONOMÉTRICA}

Essa seção irá apresentar o modelo empírico para testar a hipótese dos bens recém comercializados proposta por Baldwin \& Di Nino (2008). Conforme essa hipótese com a constituição de um acordo de integração econômica a tendência é que haja reduções nos custos fixos de entrada em um novo mercado exportador e/ou quedas nos custos variáveis do comércio e, dessa forma, algumas firmas que antes não participavam do mercado externo passarão a exportar. Intuitivamente parece lógico que uma forma de

\footnotetext{
${ }^{7}$ Em experimentos naturais sempre haverá um grupo de tratamento, que foi afetado pelo evento, quanto um grupo de controle, não afetado pela mudança. Sendo que ambos apresentam características semelhantes.

${ }^{8}$ Os não-membros são representados pelo grupo de controle, ou seja, Bolívia, Chile e Peru.
} 
Figura 3. Evolução dos zeros (base 1991).

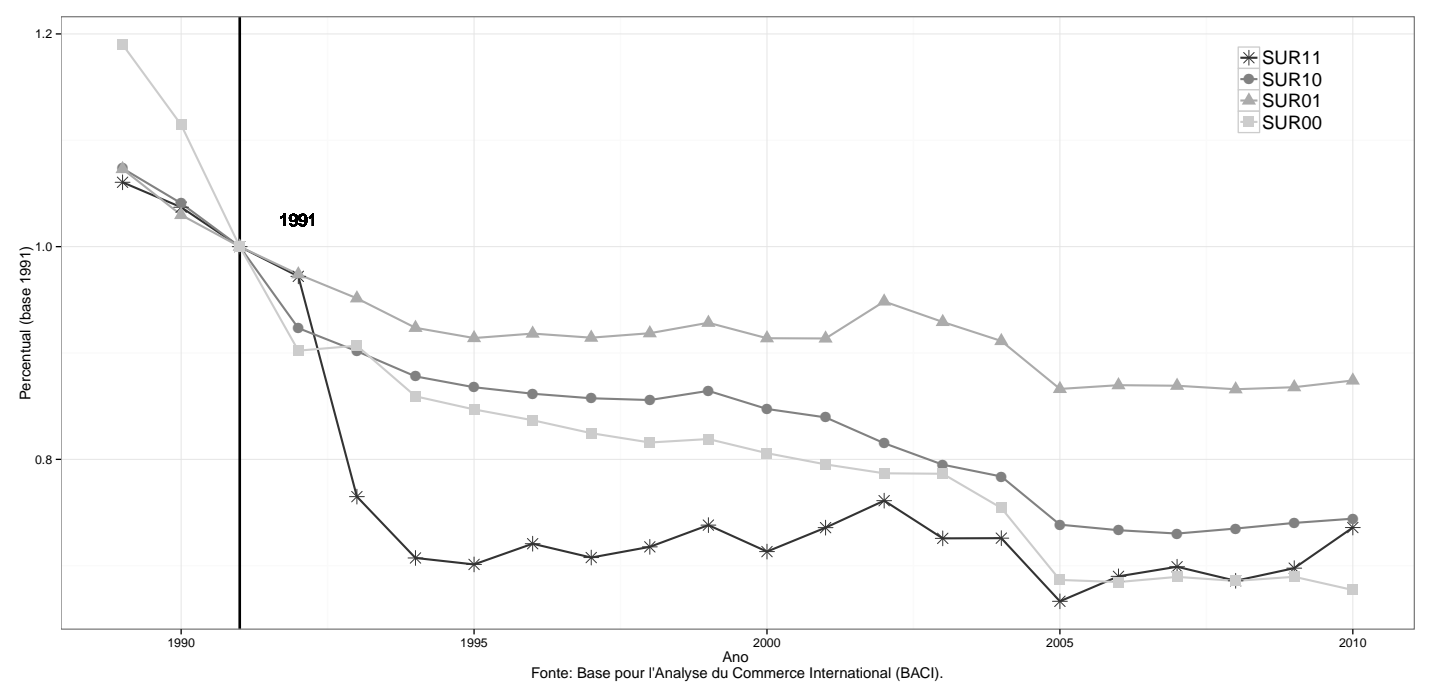

testar essa hipótese é estimar o impacto da adoção do Mercosul sobre a probabilidade de que uma nova categoria de bens seja exportada. Para calcular essa probabilidade optou-se pelo modelo de resposta qualitativa Logit. Como variável dependente, $x_{i j}$, considerou-se um para o comércio bilateral positivo e zero caso contrário.

Seguindo uma estratégia similar a de Santos Silva \& Tenreyro (2010), serão considerados dois grupos de países em cada estimação. Um grupo de tratamento, composto pelos membros plenos do Mercosul: Brasil, Argentina, Paraguai, Uruguai e Venezuela e; um grupo de controle, composto por: Bolívia, Chile e Peru. ${ }^{9}$ Como medida de robustez, foram adicionadas estimativas considerando um segundo grupo de controle composto exclusivamente por países desenvolvidos (Alemanha, Estados Unidos e Holanda).

O modelo inclui uma variável dummy denominada Laço Comercial. Ela identifica o grupo de tratamento, Mercosul, durante todo o período de análise. Como explicado em Santos Silva \& Tenreyro (2010), essa variável serve para controlar uma possível endogeneidade oriunda de características não observáveis entre os pares de países pertencentes ao Mercosul e os demais países pertencentes ao grupo de controle. A variável de interesse será a dummy de Mercosul com valor um para os membros plenos do bloco e zero caso contrário, a partir do ano da entrada em vigor do bloco (1991-2010). Também serão consideradas variáveis tradicionais dos modelos gravitacionais: o logaritmo da distância, $\log \left(\right.$ Distância $\left._{i j}\right)$, entre os parceiros comerciais para avaliar o impacto do custo de transporte sobre o comércio internacional, uma dummy Fronteira ${ }_{i j}$ com um para países que são fronteiriços e zero se não compartilham de uma mesma fronteira e uma dummy $\operatorname{Idioma}_{i j}$ que assume um se o parceiro comercial possui o mesmo idioma e zero caso contrário.

Como proxy para os índices de preços, os termos de resistências multilaterais (TRMs) que não são observáveis, também foram incluídos como regressores dummies de efeito fixo: ${ }^{10}$ um vetor $\varrho_{i, t}$ representando dummies variantes no tempo para países de origem e um vetor $\varsigma_{j, t}$ caracterizando dummies variantes no tempo para países de destino, ou seja, controlando respectivamente para as resistências multilaterais externas e internas. Por fim, foi introduzido um termo de erro $\varepsilon_{i j}$. Logo a equação repre-

\footnotetext{
${ }^{9}$ Esses três países são Estados parte da Comunidade Andina.

${ }^{10}$ Segundo Behar \& Nelson (2014) o uso de efeitos fixos exportador e importador nas estimativas é reconhecido na literatura empírica gravitacional como o melhor método no contexto das equações gravitacionais.
} 
sentativa da regressão do modelo Logit é dada por

$$
x_{i j}=\beta_{0}+\ln t_{i j}+\varrho_{i, t}+\varsigma_{j, t}+\varepsilon_{i j},
$$

em que

$$
t_{i j} \equiv d_{i j}^{\beta_{1}} \cdot \exp \left(\beta_{2} \text { Mercosul }_{i j}+\beta_{3}{\text { Laço } \left.\text { Comercial }_{i j}+\beta_{4} \text { Fronteira }_{i j}+\beta_{5} \text { Idioma }_{i j}\right)} .\right.
$$

Assim, $t_{i j}$ representa as barreiras comerciais entre $i$ e $j$ sendo que todas essas variáveis têm se mostrado significativas na determinação do comércio bilateral.

Por fim, como já destacado, assim como a COMTRADE, a BACI não registra as categorias cujo fluxo de comércio foi igual a "zero", sendo esses obtidos através da expansão dos dados iniciais. Sendo assim, para os oito países da amostra haviam inicialmente 453.464 observações sobre comércio bilateral e após a expansão o número de observações totalizou 1.527.680, ou seja, 70,3\% das observações são "zeros". Com a substituição dos TRMs pelas dummies variantes e invariantes no tempo, o modelo totalizará 355 variáveis explicativas. ${ }^{11}$

\section{RESULTADOS}

Nota-se pela Tabela 1 que todos os coeficientes angulares estimados apresentaram os sinais esperados, ${ }^{12}$ conforme a teoria dos modelos gravitacionais, bem como foram estatisticamente significantes. O sinal negativo do logaritmo da distância implica que quanto maior a distância entre $i$ e $j$ menor será o comércio entre esses países. Fronteira e Idioma apresentaram sinais positivos implicando que compartilhar uma mesma fronteira e possuir o mesmo idioma, respectivamente, impulsionam o comércio bilateral. Para a variável Mercosul os resultados encontrados sugerem que a união aduaneira possui um efeito pró-comércio uma vez que o parâmetro para essa variável foi positivo e estatisticamente diferente de zero, a um nível de significância de 5\%, sendo esse efeito "puro" pois o fluxo de comércio independente do RTA foi captado por laço comercial. Todavia, observa-se que esse valor pode ter sido subestimado devido ao viés de agregação, ou seja, alguns bens recém comercializados foram em categorias para as quais já havia algumas firmas exportando e que uma base de dados em nível de firma, que é a base ideal para essa análise, poderia apresentar um valor superior.

Entretanto, como neste artigo se está interessado na probabilidade de que uma nova categoria de bens seja comercializada calcularam-se os efeitos marginais das variáveis de interesse e, assim, percebese que essa estatística após a instituição do Mercosul foi igual a 1,87\%. Esse resultado corrobora as recentes criticas ao trabalho de Rose (2000). De acordo com esse autor, uma união monetária levaria a um aumento de $235 \%$ do fluxo bilateral de comércio. Por outro lado, estudos recentes mostram que esse tipo de integração econômica tem um pequeno efeito, de 5\% a 20\% (Baldwin \& Di Nino, 2006), ou podem ser estatisticamente insignificante (Berger \& Nitsch, 2008; Figueiredo, Lima \& Schaur, 2015; Santos Silva \& Tenreyro, 2010). Destaca-se que Figueiredo et al. (2015) empregam uma abordagem quantílica servindo como um teste de robustez para trabalhos que inferiram o efeito do Euro na média condicional.

Por fim, Fernández-Val (2009) destaca que os modelos de resposta binária podem ser viesados devido ao problema dos parâmetros incidental (Wooldridge, 2005), principalmente se a heterogeneidade individual não-observável invariante no tempo não for controlada. Contudo, nos modelos estáticos os efeitos marginais possuem um viés negligenciado, ou seja, os parâmetros estimados são próximos dos seus verdadeiros valores e, portanto, o valor para Mercosul encontrado neste trabalho representa, aproximadamente, o valor assintótico uma vez que adotou-se um modelo com covariáveis exclusivamente exógenas.

\footnotetext{
${ }^{11}$ As cinco variáveis elencadas na equação (2), efeitos fixos invariantes no tempo, mais as dummies de efeito fixo variantes no tempo.

${ }^{12}$ Por brevidade apresentam-se apenas os resultados para as variáveis da equação (2).
} 
Tabela 1. Hipótese dos bens recém comercializados.

\begin{tabular}{|c|c|c|c|c|}
\hline \multirow[b]{2}{*}{ Variáveis } & \multicolumn{2}{|c|}{ Grupo de Controle 1} & \multicolumn{2}{|c|}{ Grupo de Controle 2} \\
\hline & Coeficiente & Efeito Marginal & Coeficiente & Efeito Marginal \\
\hline \multirow[t]{2}{*}{ Mercosul } & $0,1105^{\mathrm{b}}$ & $0,0187^{b}$ & $0,2508^{\mathfrak{a}}$ & $0,0611^{\mathfrak{a}}$ \\
\hline & $(0,0510)$ & $(0,0088)$ & $(0,0349)$ & $(0,0086)$ \\
\hline \multirow[t]{2}{*}{ Laço Comercial } & $1,2782^{\mathfrak{a}}$ & $0,2528^{\mathfrak{a}}$ & $1,1518^{\mathfrak{a}}$ & $0,2800^{\mathfrak{a}}$ \\
\hline & $(0,0504)$ & $(0,0112)$ & $(0,0376)$ & $(0,0088)$ \\
\hline \multirow[t]{2}{*}{$\log ($ Distância $)$} & $-0,2870^{\mathfrak{a}}$ & $-0,0477^{\mathfrak{a}}$ & $-0,5274^{\mathfrak{a}}$ & $-0,1270^{\mathfrak{a}}$ \\
\hline & $(0,0059)$ & $(0,0010)$ & $(0,0037)$ & $(0,0009)$ \\
\hline \multirow[t]{2}{*}{ Fronteira } & $0,1803^{\mathfrak{a}}$ & $0,0307^{\mathfrak{a}}$ & $-0,5649^{\mathfrak{a}}$ & $-0,1297^{\mathfrak{a}}$ \\
\hline & $(0,0093)$ & $(0,0016)$ & $(0,0157)$ & $(0,0034)$ \\
\hline \multirow[t]{2}{*}{ Idioma } & $0,8624^{\mathfrak{a}}$ & $0,1410^{\mathfrak{a}}$ & $0,1267^{\mathfrak{a}}$ & $0,0308^{\mathfrak{a}}$ \\
\hline & $(0,0143)$ & $(0,0023)$ & $(0,0124)$ & $(0,0030)$ \\
\hline \multirow[t]{2}{*}{ Constante } & $-3,6037^{\mathfrak{a}}$ & - & $0,3072^{\mathfrak{a}}$ & - \\
\hline & $(0,1029)$ & - & $(0,0674)$ & - \\
\hline Count $R^{2}$ & \multicolumn{2}{|c|}{$78,43 \%$} & \multicolumn{2}{|c|}{$77,85 \%$} \\
\hline Amostra & \multicolumn{2}{|c|}{1.527 .680} & \multicolumn{2}{|c|}{1.528 .912} \\
\hline
\end{tabular}

Notas: Erros padrões entre parênteses. Significância estatística: ${ }^{a} 1 \%,{ }^{b} 5 \%$ e ${ }^{c} 10 \%$.

\subsection{Resultados por países}

Quem mais se beneficiou com o Mercosul? Como observamos na seção 2 o Brasil já apresentava uma maior diversidade de bens exportados antes mesmo da criação do bloco e, sendo assim, espera-se que apresente a menor probabilidade quanto a uma nova categoria de produtos em ser comercializada. Todavia, nota-se pela Tabela 2 que a Venezuela apresentou a menor probabilidade entre todos os países. Mas importante salientar que esse país tornou-se membro pleno do Mercosul apenas em 2012 além de estar atravessando uma situação política e econômica conturbada, situações que podem ter inviabilizado obter ganhos advindos da adesão ao bloco econômico. Quanto aos resultados para Brasil e Argentina destaca-se a pequena diferença na quantidade de categorias comercializadas por esses países refletindo numa homogeneidade em seus efeitos marginais.

Já com relação ao Paraguai e Uruguai esses dois membros apresentavam os menores números de categorias comercializadas em 1991, respectivamente, 249 e 505. Portanto espera-se que ambos possuam a maior probabilidade de novas categorias de serem comercializadas. 0 que pode ser comprovada através da Tabela 2 em que os valores reportados para esses dois países foram, respectivamente, 8,90\% e 11,58\%. Portanto, pelos resultados individualizados nota-se que o país mais beneficiado com a constituição do Mercosul foi o Uruguai enquanto a Argentina obteve o menor ganho. Sendo assim, esses resultados ratificam a hipótese da seção 2 de que países com uma pauta de exportação mais diversificada terão um impacto menor sobre a margem extensiva de comércio como consequência da constituição de um RTA.

\section{CONCLUSÃO}

Este artigo testou a hipótese dos bens recém comercializados proposta por Baldwin \& Di Nino (2008), no período 1989-2010, para os Estados membros plenos do Mercosul. Para tanto, considerou-se 
Tabela 2. Impactos individualizados por país.

\begin{tabular}{lcc}
\hline País & Coeficiente & Efeito Marginal \\
\hline Brasil & $0,3211^{\mathfrak{a}}$ & $0,0578^{\mathfrak{a}}$ \\
& $(0,0197)$ & $(0,0038)$ \\
Argentina & $0,2645^{\mathfrak{a}}$ & $0,0470^{\mathfrak{a}}$ \\
& $(0,0181)$ & $(0,0034)$ \\
Paraguai & $0,4781^{\mathfrak{a}}$ & $0,0890^{\mathfrak{a}}$ \\
& $(0,0228)$ & $(0,0047)$ \\
Uruguai & $0,6060^{\mathfrak{a}}$ & $0,1158^{\mathfrak{a}}$ \\
& $(0,0201)$ & $(0,0043)$ \\
Venezuela & $0,2518^{\mathfrak{a}}$ & $0,0446^{\mathfrak{a}}$ \\
& $(0,0179)$ & $(0,0033)$ \\
\hline
\end{tabular}

Notas: Erros padrões entre parênteses. Significância estatística: ${ }^{a} 1 \%,{ }^{b} 5 \%$ e ${ }^{\mathrm{c}} 10 \%$.

um banco de dados com elevado nível de desagregação. Uma vez que o banco de dados só reporta as informações positivas (existência) de comércio, foi necessário fazer uma expansão da base de modo a refletir os fluxos bilaterais de comércio iguais a "zero". A abordagem econométrica empregada foi um estimador Logit cuja variável de resposta, $x_{i j}$, assume um para o comércio bilateral positivo e zero caso contrário. Como regressores utilizaram-se as variáveis comum na literatura gravitacional: i) logaritmo da distância, ii) fronteira, iii) idioma, iv) Mercosul (caracterizando o RTA) e v) laço comercial (representando a aproximação comercial entre os países), além das dummies de efeito fixo variantes e invariantes no tempo.

A especificação principal indicou que o Mercosul aumentou a probabilidade de entrada de novos produtos na pauta de exportação. Porém, os resultados apresentam algumas heterogeneidades: o Uruguai foi o mais beneficiado pelo acordo enquanto a Argentina foi o que obteve o menor ganho proveniente da integração econômica.

Por fim, recomenda-se a aplicação de modelos estruturais, tais como o de Anderson, Larch \& Yotov (2014), em trabalhos futuros. Tal metodologia tornaria possível a criação de cenários contrafactuais o que permitiria responder quais os custos de oportunidades associados à estratégia comercial brasileira voltada quase que exclusivamente ao Mercosul.

\section{REFERÊNCIAS BIBLIOGRÁFICAS}

Anderson, J. E., Larch, M. \& Yotov, Y. V. (2014). Growth and trade: A structural approach [Mimeo].

Baier, S. L., \& Bergstrand, J. H. (2007). Do free trade agreements actually increase members' international trade? Journal of International Economics, 71(1), 72-95. doi: 10.1016/j.jinteco.2006.02.005

Baier, S. L., Bergstrand, J. H. \& Feng, M. (2013). Economic integration agreements and the margins of international trade. Journal of International Economics, 93(2), 339-350. doi: 10.1016/j.jinteco.2014.03.005

Baldwin, R.E., \& Di Nino, V. (2006, November). Euros and zeros: The common currency effect on trade in new goods (Working Paper $\mathrm{N}^{0}$ 12673). National Bureau of Economic Research (NBER). doi: 10.3386/w12673

Baldwin, R.E., Di Nino, V., Fontagné, L., De Santis, R. A. \& Taglioni, D. (2008). Study on the impact of the Euro on trade and foreign direct investment (Economic Papers $N^{0}$ 321). Brussels: European Commission. doi: 


\section{$10.2765 / 65274$}

Baldwin, R.E., \& Di Nino, V. (2008). The newly-traded goods hypothesis: Evidence from the trade data. In R. E. Baldwin, V. Di Nino, L. Fontagne, R. A. De Santis \& D. Taglioni (Eds.), Study on the impact of the Euro on trade and foreign direct investment. SSRN Electronic Journal. doi: 10.2139/ssrn.1163774

Baltagi, B. H., Egger, P. \& Pfaffermayr, M. (2008). Estimating regional trade agreement effects on FDI in an interdependent world. Journal of Econometrics, 145(1-2), 194-208. doi: 10.1016/j.jeconom.2008.05.017

Behar, A., \& Nelson, B. D. (2014). Trade flows, multilateral resistance, and firm heterogeneity. Review of Economics and Statistics, 96(3), 538-549. doi: 10.1162/REST_a_00380

Berger, H., \& Nitsch, V. (2008). Zooming out: The trade effect of the euro in historical perspective. Journal of International Money and Finance, 27(8), 1244-1260. doi: 10.1016/j.jimonfin.2008.07.005

Chaney, T. (2008). Distorted gravity: The intensive and extensive margins of international trade. American Economic Review, 98(4), 1707-1721. doi: 10.1257/aer.98.4.1707

Dutt, P., Mihov, I. \& Van Zandt, T. (2013). The effect of WTO on the extensive and the intensive margins of trade. Journal of International Economics, 91(2), 204-219. doi: 10.1016/j.jintec0.2013.08.001

Fernández-Val, I. (2009). Fixed effects estimation of structural parameters and marginal effects in panel probit models. Journal of Econometrics, 150(1), 71-85. doi: 10.1016/j.jeconom.2009.02.007

Figueiredo, E., Lima, L. R. \& Schaur, G. (2015). The effect of the Euro on the bilateral trade distribution. Empirical Economics, 50(1), 17-29. doi: 10.1007/s00181-015-1004-1

Gaulier, G., \& Zignago, S. (2010). BACI: International trade database at the product-level (The 1994-2007 version) (Working Paper/Document de Travail N ${ }^{0}$ 2010-23). Centre d'Études Prospectives et d'Informations Internationales - CEPII. doi: 10.2139/ssrn.1994500

Melitz, M.J. (2003). The impact of trade on intra-industry reallocations and aggregate industry productivity. Econometrica, 71(6), 1695-1725. doi: 10.1111/1468-0262.00467

Orefice, G. (2015). International migration and trade agreements: The new role of PTAs. Canadian Journal of Economics, 48(1), 310-334. doi: 10.1111/caje.12122

Rose, A. (2000). One money, one market: Estimating the effect of common currencies on trade. Economic Policy, 15(30), 08-45. doi: 10.1111/1468-0327.00056

Santos Silva, J., \& Tenreyro, S. (2010). Currency unions in prospect and retrospect. Annual Review of Economics, 2(1), 51-74. doi: 10.1146/annurev.economics.102308.124508

Wooldridge, J. M. (2005). Simple solutions to the initial conditions problem in dynamic, nonlinear panel data models with unobserved heterogeneity.Journal of Applied Econometrics, 20(1), 39-54. doi: 10.1002/jae.770 